\title{
Child Pugh and ALBI grade: past, present or future?
}

\author{
Margherita Rimini, Giulia Rovesti, Andrea Casadei-Gardini \\ Department of Oncology and Hematology, Division of Oncology, University of Modena and Reggio Emilia, Modena, Italy \\ Correspondence to: Andrea Casadei-Gardini, MD. Department of Oncology and Hematology, Division of Oncology; University Hospital of Modena, \\ Modena, Via Del Pozzo 72, 41124, Italy. Email: casadeigardini@gmail.com. \\ Provenance and Peer Review: This article was commissioned by the editorial office, Annals of Translational Medicine. The article did not undergo \\ external peer review. \\ Comment on: Feng D, Wang M, Hu J, et al. Prognostic value of the albumin-bilirubin grade in patients with hepatocellular carcinoma and other liver \\ diseases. Ann Transl Med 2020;8:553.
}

Submitted May 06, 2020. Accepted for publication May 21, 2020.

doi: $10.21037 /$ atm-20-3709

View this article at: http://dx.doi.org/10.21037/atm-20-3709

Hepatocellular carcinoma (HCC) is the most common primary liver malignancy, and it has been reported to be the fifth-most common cancer worldwide and the third leading cause of cancer-related death (1). The unique nature of this kind of malignancy, which in the vast majority of cases occurs in patients with underlying advanced liver disease, makes the decision-making process a real global health challenge, as it must take in count the tumor characteristics, the liver function and the physical status. In last decades, many efforts were made in order to expand the therapeutic armamentarium for this disease, in both locoregional and systemic treatments settings, and to find new prognostic indexes able to stratify patients and improve outcomes. Historically, the Child-Pugh (CP) score system has been elected as tool for liver assessment and it has been integrated in the guidelines for the management of HCC. More recently, the albumin-bilirubin (ALBI) grade has been proposed as an alternative nomogram to evaluate the liver impairment for these patients.

Feng et al. critically analyzed the role of ALBI grade in HCC, on the basis of the data available to date in literature. First of all, they discussed the advantages and the shortcomings of both liver assessment tools, the CP score system and the ALBI grade, highlighting the major objectivity that characterizes the second one. The authors commented that ALBI score has demonstrated to improve the accuracy of prognoses, especially within the CP A group, being a more suitable tool of liver function assessment (2,3). For this reason, the ALBI grade has been tested in association with the BCLC stage, showing a clinically significant stratifying power across both curative and palliative stages (4); in the same way, the ALBI score has successfully replaced the CP scoring system in assessing the liver function in other HCC staging systems. They concluded by mentioning the need of more high-level evidences to deepen the pending issues, including the lack of portal hypertension parameters in the ALBI grade, and its prognostic value in the post-treatment setting.

Our opinion about the prognostic role and the stratification power of ALBI grade compared to CP score system is strictly in line with what discussed by colleagues.

HCC is a complex disease, which in the vast majority of cases arises as complication of liver chronic disease, thus making the prognostic interaction between tumor characteristics and liver residual function the primary challenge in the process of decision making of this disease.

Several staging systems have been proposed, but none of them has been universally accepted. Currently, the most used staging systems in HCC are the BCLC system (5), the Cancer of the Liver Italian program (CLIP) system (6) and the Japanese staging (JIS) system (7); all of these include the liver function assessment according to the CP score, which however has been highlighted to present some shortcomings.

First of all, since it includes ascites and encephalopathy, which both depend on the clinician's judgment, CP score is subjective. Secondly, interrelation between some of the parameters in the CP score system, including ascites and albumin, is close. Thirdly, CP score system was originally developed as an empirical method for the prognostic 
stratification of patients affected by bleeding complications of cirrhosis and portal hypertension (8), thus showing a lack of reproducibility for the liver function assessment in HCC patients with a non-cirrhotic background.

The acknowledged clinical heterogeneity of HCC suggests the urgent need for objective, reproducible and accurate prognostic biomarkers to surpass the subjectivityderived bias of the $\mathrm{CP}$ score, in order to better assess the liver function and to improve patient outcomes.

The ALBI grade is an objective nomogram established through an evidence-based process. It is constituted by two accessible biochemical parameters, albumin and bilirubin, which makes the score simple, economic and likely to be integrated in staging systems. As reported by Feng et al., the prognostic power of ALBI grade has been highlighted in many studies, regardless of the therapeutic option and in both curative and palliative settings (2,9-11).

In our previous study conducted on a real-life cohort of 398 HCC patients treated with sorafenib, the ALBI grade confirmed a stronger role in evaluating hepatic impairment if compared to the $\mathrm{CP}$ score system; in multivariate analysis, in fact, the prediction power constantly remained for the ALBI grade, while disappeared for the CP score (12). Regarding the two factors comprised in the ALBI score, both albumin and bilirubin were identified as potential prognostic factors, but in the multivariate analysis context only albumin $<35 \mathrm{~g} / \mathrm{L}$ emerged as an independent prognostic factor for poorer OS, thus showing that albumin, rather than bilirubin, is the most relevant factor able to make the ALBI grade the strongest prognostic factor (12). In the CP scoring system, the albumin prognostic power could be flattened by the close connection with others parameters, such as ascites, as we discussed above.

On the other hand, it is necessary to mention ALBI grade drawbacks, wondering if it is possible to increase its efficacy.

Primarily, all the data available about HCC are based on the liver function assessment according to the CP score, since it has been adopted into inclusion and exclusion criteria in the majority of clinical trials, while ALBI grade has been used only as stratification factor; new prospective trials stratifying patients on the basis of the ALBI grade are needed in order to confirm its prognostic efficacy. Secondly, if, on one hand, it has been demonstrated that the ALBI grade has a predictive power for assessing liver function higher than that of CP score, on the other hand, by retrospectively applying the ALBI grade, this threesided score turned out to be basically a two-sided score in particularly in patients with CP-A-B7. In fact, all HCC trials included patients eligible for liver resection, locoregional treatments and pharmacological treatments, on the basis of a good liver function expressed as CP score A or B7, which correspond to ALBI grade 1 or 2; data about patients with ALBI grade 3 are still scarce.

Thirdly, the ALBI score does not include platelet counts or other biomarkers related to portal hypertension, as well as inflammation status. The importance of portal hypertension has emerged from the BCLC prognostic algorithm, which includes the invasive measurement of hepatic venous pressure gradients for patients who are considered for radical treatments (5). In the study of Liu et al., the PALBI grade, obtained by the integration of ALBI grade with the platelet count, demonstrated to adequately predict survivors across various HCC stages (13).

Concerning the inflammation status, there is a strong rationale for the integration of inflammatory indexes into prognostic scores in HCC. Chronic inflammation, characterized by infiltration of macrophages, immature myeloid cells and, consequently, by an altered production of cytokines, may be considered to be one of the most important triggers of HCC carcinogenesis and tumor progression (14). In fact, the vast majority of HCC cases rises on a background of chronic hepatitis or cirrhosis, thus it is not surprising that inflammatory indexes could be associated with the HCC prognosis; moreover, some inflammatory indexes include indirect markers of liver function, such as platelet count.

In the last years, several inflammation prognostic scores, such as the systemic immune-inflammation index (SII), the neutrophil to lymphocyte ratio (NLR), the platelet to lymphocyte ratio (PLR) and the aspartate aminotransferase (AST) to lymphocyte ratio (ALRI) showed to predict survival and recurrence of HCC patients in both curative and palliative settings $(15,16)$, suggesting that the integration between liver function biomarkers and inflammatory indexes could allow a better stratification of patients.

In a previous work, our group analyzed a real-life cohort of $\mathrm{HCV}$ patients treated with direct-acting antiviral therapies (DAAs), founding that an impaired ALBI score is an independent risk factor for the occurrence of HCC, while the ALRI index predict the recurrence of cancer in patients with a history of HCC (17). In another study, analyzing a cohort of patients with advanced HCC treated with Sorafenib, Rovesti et al. confirmed at the multivariate analysis that PLR and SII are prognostic factors for OS (12). 
We conclude that the prognostic power of the ALBI grade could be improved by the integration of data about inflammation status; PLR and SII are likely to be the most indicative, since they include platelet, lymphocytes and neutrophil counts, all of which correlate with tumor development, progression and, if considering the platelets count, with liver function (18).

In the perspective of finding alternative and non-invasive strategies to assess liver function, the research landscape is moving towards the computational medical imaging, known as Radiomics, which allow a high-throughput extraction of features from medical images (e.g., CTs, RMNs, PETs) $(19,20)$.

Through the texture analysis, the Radiomics technique scan captures high-dimensional data concerning cancers' pathological and molecular phenotypes, tumor microenvironment and other features with prognostic and clinical significance, thus helping in diagnosis and treatment decision making processes. Compared to needle biopsies, Radiomics has the advantage of being non-invasive, since it is based on the analysis of CT/RMN images, which are normally available across all phases of the disease, from diagnosis to the end of treatment.

In HCC field, Radiomics has found a role in diagnosis, in the classification of focal liver lesions (21), in the prediction of response to treatments, both surgical and medical $(22,23)$, in prognostic evaluation and microvascular infiltration detection (24) and in gene phenotype profiling (25). The use of Radiomics as a tool to assess HCC liver function has not been explored yet.

Radiomics is a resource still far from being fully exploited but it is plausible that information about liver function will soon be available through the texture analysis; such information, once validated, could be integrated with biochemical parameters included in the ALBI grade, in order to build more comprehensive scores able to assess liver function and tumor extension across spatial-temporal limitations.

In conclusion, the CP score system and the ALBI grade are the tools we are currently using to assess liver impairment in HCC patients, but both present some shortcomings. The new insights in the interplay between cancer and inflammation and the new imaging technologies could provide us the pieces we were missing to improve our prognostic scores and better stratify patients in the decisionmaking process of this complex disease.

\section{Acknowledgments}

Funding: None.

\section{Footnote}

Conflicts of Interest: All authors have completed the ICMJE uniform disclosure form (available at http://dx.doi. org/10.21037/atm-20-3709). The authors have no conflicts of interests to declare.

Etbical Statement: The authors are accountable for all aspects of the work in ensuring that questions related to the accuracy or integrity of any part of the work are appropriately investigated and resolved.

Open Access Statement: This is an Open Access article distributed in accordance with the Creative Commons Attribution-NonCommercial-NoDerivs 4.0 International License (CC BY-NC-ND 4.0), which permits the noncommercial replication and distribution of the article with the strict proviso that no changes or edits are made and the original work is properly cited (including links to both the formal publication through the relevant DOI and the license). See: https://creativecommons.org/licenses/by-nc-nd/4.0/.

\section{References}

1. Ferlay J, Shin HR, Bray F, et al. Estimates of worldwide burden of cancer in 2008: GLOBOCAN 2008. Int J Cancer 2010;127:2893-917.

2. Pinato DJ, Yen C, Bettinger D, et al. The albumin bilirubin grade improves hepatic reserve estimation post sorafenib failure: implications for drug development. Aliment Pharmacol Ther 2017;45:714-22.

3. Waked I, Berhane S, Toyoda H, et al. Transarterial chemo-embolisation of hepatocellular carcinoma: impact of liver function and vascular invasion. Br J Cancer 2017;116:448-54.

4. Pinato DJ, Sharma R, Allara E, et al. The ALBI grade provides objective hepatic reserve estimation across each BCLC stage of hepatocellular carcinoma. J Hepatol 2017;66:338-46.

5. Llovet JM, Brú C, Bruix J. Prognosis of hepatocellular carcinoma: the BCLC staging classification. Semin Liver Dis 1999;19:329-38.

6. A new prognostic system for hepatocellular carcinoma: a retrospective study of 435 patients: the Cancer of the Liver Italian Program (CLIP) investigators. Hepatology 1998;28:751-5.

7. Kudo M, Chung H, Osaki Y, et al. Prognostic staging system for hepatocellular carcinoma CLIP score): its 
value and limitations, and a proposal for a new staging system, the Japan Integrated Staging Score JIS score. J Gastroenterol 2003;38:207-15.

8. Child CG, Turcotte JG. Surgery and portal hypertension. Major Probl Clin Surg 1964;1:1-85.

9. Toesca DAS, Osmundson EC, von Eyben R, et al. Assessment of hepatic function decline after stereotactic body radiation therapy for primary liver cancer. Pract Radiat Oncol 2017;7:173-82.

10. Ma XL, Zhou JY, Gao XH, et al. Application of the albumin-bilirubin grade for predicting prognosis after curative resection of patients with early-stage hepatocellular carcinoma. Clin Chim Acta 2016;462:15-22.

11. Kao WY, Su CW, Chiou YY, et al. Hepatocellular Carcinoma: Nomograms Based on the Albumin-Bilirubin Grade to Assess the Outcomes of Radiofrequency Ablation. Radiology 2017;285:670-80.

12. Rovesti G, Orsi G, Kalliopi A, et al. Impact of Baseline Characteristics on the Overall Survival of HCC Patients Treated with Sorafenib: Ten Years of Experience. Gastrointest Tumors 2019;6:92-107.

13. Liu PH, Hsu CY, Hsia CY, et al. ALBI and PALBI grade predict survival for HCC across treatment modalities and BCLC stages in the MELD Era. J Gastroenterol Hepatol 2017;32:879-86.

14. Refolo MG, Messa C, Guerra V, et al. Inflammatory Mechanisms of HCC Development. Cancers (Basel) 2020;12:641.

15. Wang D, Bai N, Hu X, et al. Preoperative inflammatory markers of NLR and PLR as indicators of poor prognosis in resectable HCC. Peer J 2019;7:e7132.

16. Zhao LY, Yang DD, Ma XK, et al. The Prognostic Value of aspartate aminotransferase to lymphocyte ratio and systemic immune-inflammation index for Overall Survival of Hepatocellular Carcinoma Patients Treated with palliative Treatments. J Cancer 2019;10:2299-311.

Cite this article as: Rimini M, Rovesti G, Casadei-Gardini A. Child Pugh and ALBI grade: past, present or future? Ann Transl Med 2020;8(17):1044. doi: 10.21037/atm-20-3709
17. Casadei Gardini A, Foschi FG, Conti F, et al. Immune inflammation indicators and ALBI score to predict liver cancer in $\mathrm{HCV}$-patients treated with direct-acting antivirals. Dig Liver Dis 2019;51:681-8.

18. Casadei Gardini A, Scarpi E, Faloppi L, et al. Immune inflammation indicators and implication for immune modulation strategies in advanced hepatocellular carcinoma patients receiving sorafenib. Oncotarget 2016;7:67142-9.

19. Lambin P, Rios-Velazquez E, Leijenaar R, et al. Radiomics: extracting more information from medical images using advanced feature analysis. Eur J Cancer 2012;48:441-6.

20. Aerts HJ, Velazquez ER, Leijenaar RT, et al. Decoding tumour phenotype by noninvasive imaging using a quantitative radiomics approach. Nat Commun 2014;5:4006.

21. Li Z, Mao Y, Huang W, et al. Texture-based classification of different single liver lesion based on spair T2W MRI images. BMC Med Imaging 2017;17:42.

22. Mulé S, Thiefin G, Costentin C, et al. Advanced hepatocellular carcinoma: Pretreatment contrastenhanced CT texture parameters as predictive biomarkers of survival in patients treated with sorafenib. Radiology 2018;288:445-55.

23. Akai H, Yasaka K, Kunimatsu A, et al. Predicting prognosis of resected hepatocellular carcinoma by radiomics analysis with random survival forest. Diagn Interv Imaging 2018;99:643-51.

24. Banerjee S, Wang DS, Kim HJ, et al. A computed tomography radiogenomic biomarker predicts microvascular invasion and clinical outcomes in hepatocellular carcinoma. Hepatology 2015;62:792-800.

25. Taouli B, Hoshida Y, Kakite S, et al. Imaging-based surrogate markers of transcriptome subclasses and signatures in hepatocellular carcinoma: Preliminary results. Eur Radiol 2017;27:4472-81. 